\title{
Empleo de criptógamas como herramienta ecológica de biomonitorización del depósito de nitrógeno en la península ibérica
}

\author{
Z. Varela ${ }^{1,2 *}$, S.C. Aranda ${ }^{3}$, B. Estébanez Pérez ${ }^{4}$, N.G. Medina ${ }^{4}$, M.T. Boquete ${ }^{5,6}$ \\ (1) Centre for Ecology, Evolution and Environmental Changes, Facultade de Ciências, Universidade de Lisboa, 1749-016 Lisboa, Portugal. \\ (2) Departamento de Bioloxía Funcional, Facultade de Bioloxía, Universidade de Santiago de Compostela, 15782 Santiago de Compostela, España. \\ (3) Departamento de Biología, Geología, Física y Química Inorgánica, Universidad Rey Juan Carlos, 28933 Móstoles, Madrid, España. \\ (4) Departamento de Biología, Comisión Docente de Botánica, Universidad Autónoma de Madrid, 28049 Madrid, España. \\ (5) Department of Integrative Biology, University of South Florida, 33620 Tampa, FL. Estados Unidos. \\ (6) Department of Ecology and Evolution of Plant-Animal Interactions, Estación Biológica de Doñana, CSIC, 41092 Sevilla, España.
}

*Autor de correpondencia: Z. Varela [zulema.varela@usc.es]

> Recibido el 28 de octubre de 2016 - Aceptado el 06 de marzo de 2017

\begin{abstract}
Varela, Z., Aranda, S.C., Estébanez Pérez, B., Medina, N.G., Boquete, M.T. 2017. Empleo de criptógamas como herramienta ecológica de biomonitorización del depósito de nitrógeno en la península ibérica. Ecosistemas 26(1): 45-54. Doi.: 10.7818/ECOS.2017.26-1.07

El nitrógeno $(\mathrm{N})$ es un macronutriente vital para los organismos vivos. Sin embargo, su entrada en grandes cantidades genera problemas como la eutrofización y acidificación del medio y cambios en las comunidades. El principal objetivo de esta revisión es describir los posibles efectos que produce el aumento antropogénico del $\mathrm{N}$ reactivo en las comunidades de criptógamas (briófitos y líquenes), un componente clave de muchos ecosistemas. Pretendemos, además, evaluar la utilidad de dichos organismos como biomonitores del depósito de este nutriente y contaminante en la Península ibérica. Las criptógamas son organismos sensibles al incremento de $\mathrm{N}$ en el medio, por lo que se han empleado ya con éxito en la Península ibérica como bioindicadores de la contaminación por $\mathrm{N}$. Por otro lado, son capaces de acumular N, proporcionando información sobre la superación de umbrales críticos en los ecosistemas y del origen de las emisiones del $\mathrm{N}$ depositado mediante la relación isotópica del $\mathrm{N}$ ( $\delta 15 \mathrm{~N})$. Finalmente, revisamos las principales características morfo-funcionales de los organismos mencionados, su papel en el ciclo del $\mathrm{N}$, los efectos que sufren por el depósito de $\mathrm{N}$ antropogénico, y analizamos su potencial como biomonitores, tanto en general como a través de un caso práctico desarrollado en la península ibérica empleando musgos terrestres.
\end{abstract}

Palabras clave: depósito de nitrógeno; biomonitorización; líquenes; briófitos

Varela, Z., Aranda, S.C., Estébanez Pérez, B., Medina, N.G., Boquete, M.T. 2017. The use of cryptogams as an ecological tool for nitrogen deposition biomonitoring in the Iberian Peninsula. Ecosistemas 26(1): 45-54. Doi.: 10.7818/ECOS.2017.26-1.07

Nitrogen is an important macronutrient for all living organisms. However, the increased nitrogen inputs in natural ecosystems generate diverse problems, such as eutrophication and acidification and changes in communities. The main scope of the present review is to describe the effects of the increase of the anthropogenic reactive nitrogen $(\mathrm{N})$ on communities of cryptogams (bryophytes and lichens), a key component of many ecosystems. We also aim to evaluate the utility of these organisms as biomonitors of the atmospheric deposition of this nutrient and pollutant in the Iberian Peninsula. Cryptogams are sensitive to $\mathrm{N}$ increases in the environment, and for this reason they have already been successfully used as bioindicators of $\mathrm{N}$ pollution in the Iberian Peninsula. Besides, they accumulate $\mathrm{N}$ in their tissues providing information on the surpassing of critical thresholds and on the sources of the deposited $\mathrm{N}$ by means of the $\mathrm{N}$ isotopic relationship $(\delta 15 \mathrm{~N})$. Finally, we review their main morpho-functional traits, their role in the $\mathrm{N}$ cycle, how they are affected by the deposition of anthropogenic $\mathrm{N}$, and we also analyse their potential in biomonitoring, especially through a case study in the Iberian Peninsula using terrestrial mosses.

Key words: Nitrogen deposition; biomonitoring; lichens; bryophytes

\section{Introducción}

El nitrógeno en forma gaseosa inerte $\left(\mathrm{N}_{2}\right)$ es el elemento más abundante en el aire (78\%) y, a pesar de ello, el crecimiento (producción primaria neta) de las plantas está frecuentemente limitado por la cantidad de $\mathrm{N}$ disponible en el medio (Vitousek y Field 2001). Esto es así porque los organismos fotosintéticos no son capaces de utilizar directamente el $\mathrm{N}$ en esta forma química y se ven limitados a la utilización de las formas reactivas de este elemento, cuya abundancia es muy inferior. De este modo, muchas especies de plantas se han adaptado a la baja disponibilidad de este nutriente incrementando la eficiencia en su captación y uso y, consecuentemente, desarrollando una sensibilidad al incremento de sus concentraciones en los ecosistemas (Bobbink et al. 1998). Actualmente las tasas de depósito de $\mathrm{N}$ de origen antropogénico, en torno a los $10 \mathrm{~kg} \mathrm{~N} \mathrm{ha}{ }^{-1} a_{n} o^{-1}$ en muchas regiones del mundo (Galloway et al. 2008), están un orden de magnitud por encima de los niveles naturales. Además, se predice que dichas concentraciones pueden duplicarse hacia 2050, e incluso alcanzar los $50 \mathrm{~kg} \mathrm{~N} \mathrm{ha}^{-1}$ año-1 $^{-1} \mathrm{en}$ algunas regiones (Galloway et al. 2004). Estos niveles superan claramente los umbrales críticos que alteran los procesos biológicos y geoquímicos, que se estiman normalmente en un rango de 5 a 
$20 \mathrm{~kg} \mathrm{~N} \mathrm{ha}^{-1}$ año-1 (Bobbink et al. 2010), provocando la acidificación del suelo y la eutrofización de los ecosistemas terrestres y acuáticos. Esta contaminación supone la tercera causa principal de pérdida biodiversidad global (Alkemade et al. 2009), así como importantes pérdidas económicas cuantificables para la Unión Europea en decenas de miles de millones de euros (Sutton et al. 2011a). Por este motivo, a día de hoy es de vital importancia evaluar los efectos de un depósito de $\mathrm{N}$ elevado en los diversos ecosistemas y comunidades, así como desarrollar metodologías que permitan cuantificar de forma fácil y eficaz dicho depósito, para lo cual las criptógamas han demostrado ser particularmente útiles.

Las criptógamas - particularmente briófitos y líquenes- son elementos clave en el ciclo biogeoquímico del N. De manera directa, influyen en la fijación del $\mathrm{N}$ no reactivo a través de su asociación con comunidades de cianobacterias (integrantes del talo de algunos líquenes o asociadas a diversos briófitos), y modifican las formas químicas en que el $\mathrm{N}$ reactivo está disponible para los restantes componentes de la biosfera, sea como ion nitrato $\left(\mathrm{NO}_{3}{ }^{-}\right)$, ion amonio $\left(\mathrm{NH}_{4}{ }^{+}\right)$, o compuestos orgánicos nitrogenados. De manera indirecta, destaca su influencia, por un lado, estabilizando los suelos, con lo que permiten la retención en cierta medida del $\mathrm{N}$ reactivo (en general altamente soluble); $y$, por otro, favoreciendo el desarrollo de otros organismos. De ambas formas ejercen efectos reseñables sobre las redes tróficas y sobre el flujo de nutrientes en el ecosistema. Además, la amplia distribución geográfica de muchas especies, con frecuencia incluso cosmopolitas, permite estudiar los patrones espacio-temporales del depósito atmosférico del $\mathrm{N}$ y promete aportar datos reveladores a escalas superiores, según apuntan los modelos existentes (Sutton et al. 2011a). Así, en la actualidad es bastante frecuente incluir estos organismos como modelo en estudios científicos (véase, por ejemplo, Sheppard et al. 2011, o Stevens et al. 2016). En el caso de la península ibérica, el número de publicaciones en este campo ha incrementado notablemente en los últimos 10 años, tanto en el área de ciencia básica con especial atención al funcionamiento ecosistémico (e.g., Maestre et al. 2011; Delgado-Baquerizo et al. 2014), como en ciencia aplicada en relación a la biomonitorización (González-Miqueo et al. 2009, 2010a, 2010b; Varela et al. 2013, 2016). A continuación, describiremos el rol de briófitos y líquenes en el ciclo biogeoquímico del $\mathrm{N}$, los posibles efectos negativos del aumento del depósito de $\mathrm{N}$ antropogénico en las comunidades de criptógamas, y su potencial como biomonitores del depósito de este nutriente y contaminante. Presentamos, además, un caso práctico llevado a cabo en Galicia, noroeste de la península ibérica. En este caso práctico se evalúa, por un lado, la variación espacial sistemática a escala regional de las concentraciones de $\mathrm{N}$ total y la relación isotópica del $\mathrm{N}\left(\delta^{15} \mathrm{~N}\right)$ en el musgo terrestre Pseudoscleropodium purum. Por otro lado, se estudia la distribución espacial de $\mathrm{N}$ total en muestras de la misma especie en los alrededores de un foco de emisión de $\mathrm{NO}_{x}$ con el objetivo de identificar las ventajas y limitaciones de estos organismos como biomonitores del depósito de $\mathrm{N}$.

\section{Briófitos y líquenes: introducción y características morfo-funcionales}

Los briófitos, con unas 16000 especies (Shaw et al. 2011), son plantas terrestres caracterizadas por poseer un desarrollo y complejidad de tejidos menor que las plantas vasculares y a su vez, por la sencillez de su fase esporofítica, que nunca se ramifica, y que produce un único esporangio. Por este motivo son consideradas como plantas no vasculares. Este grupo, que engloba a los musgos, hepáticas y antocerotas, presenta dos tipos morfológicos principales: laminares o talosos (con un cuerpo vegetativo normalmente dorsiventral), y foliosos (con un cuerpo organizado en ejes similares a tallos, llamados caulidios, y expansiones fotosintéticas parecidas a hojas, llamadas filidios).

Por su parte, los líquenes agrupan algo más de 19000 especies conocidas (Lucking et al. 2016), aunque se estima que su diversidad puede superar 28000 especies (Zedda y Rambold 2015). Se consideran tradicionalmente como una forma de vida combinada, resultante de la asociación simbiótica de un componente fúngico, o micobionte, y un componente fotosintético, o fotobionte, correspondiente a cianobacterias o algas eucariotas. Sin embargo, recientemente se ha considerado la presencia de un tercer componente en la simbiosis: una levadura de la división Basidiomycota presente en numerosas especies de líquenes (Spribille et al. 2016). Con tanta heterogeneidad de organismos, el fenotipo resultante es un talo de morfologías variadas, considerándose tres tipos fundamentales: crustáceos (forma de costra y fuertemente adheridos al sustrato por su cara inferior), foliáceos (forma de hojas o láminas, con un borde que se separa bien del sustrato) y fruticulosos (forma de pequeños arbustos y sujetos al sustrato por un único punto).

Las principales características ecofisiológicas de ambos grupos de organismos son su estrategia típicamente poiquilohidríca, es decir, son susceptibles de sufrir una rápida deshidratación en condiciones de baja humedad relativa (pasando a estado de latencia) y la reviviscencia, ya que son capaces de reanudar rápidamente la actividad metabólica tras la rehidratación (Alpert y Oliver 2002). Ambos grupos carecen de sistema radicular bien desarrollado y su cutícula, de estar presente, es muy fina (a diferencia del resto de plantas terrestres), por lo que obtienen el agua y los nutrientes directamente de la atmósfera a través de toda su superficie. Todas estas características les proporcionan la capacidad de superar condiciones desfavorables, por lo que aparecen en casi todos los ecosistemas terrestres, incluyendo diversos extremos de temperatura y humedad (Cornelissen et al. 2007). En el caso de los líquenes, pueden colonizar incluso ambientes marinos (Jones et al. 2009) o hábitats endolíticos en las zonas más inhóspitas, por frío y desecación, de la Antártida continental (de los Ríos et al. 2005).

\section{Briófitos y líquenes en el ciclo biogeoquímico del $\mathbf{N}$}

En relación con el metabolismo del $\mathrm{N}$, muchos representantes de ambos grupos pueden considerarse como frugales para este y otros nutrientes, aunque también hay especies claramente nitrófilas, como la mayoría de los musgos de la familia Splachnaceae (que viven sobre estiércol o cadáveres), o como el liquen Xanthoria parietina (propio de ambientes ricos en N). Sin embargo, en general, la cantidad de N (y P) presentes en sus cuerpos vegetativos no se comporta como un buen predictor de su crecimiento y actividad fotosintética (Cornelissen et al. 2007). Al igual que las plantas vasculares, los líquenes y los briófitos pueden tomar el $\mathrm{N}$ como $\mathrm{NO}_{3}$ (forma oxidada) o como $\mathrm{NH}_{4}^{+}$(forma reducida). A pesar de las variaciones entre especies y entre hábitats, estos grupos, con respecto de las plantas vasculares, muestran una mayor eficiencia en la toma de $\mathrm{NH}_{4}{ }^{+}$frente a la de $\mathrm{NO}_{3}{ }^{-}$(Glime 2007; Hauck 2010).

No obstante, estos grupos de criptógamas presentan opciones adicionales para absorber este nutriente: los cianolíquenes (líquenes cuyo fotobionte son las cianobacterias), y los briófitos son capaces de albergar cianobacterias fijadoras (Adams y Duggan 2008), ya sea facultativamente, como epífitas, o bien en cavidades, como muchas antocerotas, algunas hepáticas, o musgos del género Sphagnum (presentes en las turberas), cuyas células muertas y vacías (hialocistes) contienen comunidades muy complejas de microorganismos (During y Van Tooren 1990; Mitchell et al. 2003), y son capaces de aprovechar el $\mathrm{N}$ atmosférico que estas bacterias fijan. Además, pueden tomar $\mathrm{N}$ reactivo absorbiendo moléculas orgánicas, como los aminoácidos, por lo que pueden beneficiarse de la descomposición de otros organismos del ecosistema, en una escala que aún no es del todo conocida en condiciones naturales (Glime 2007; Krab et al. 2008; Hauck 2010).

Tanto los briófitos como los líquenes juegan un papel esencial en la retención de nutrientes, especialmente del N, en el mantenimiento de la estabilidad hídrica y térmica, y en la formación de sustratos utilizables por plantas vasculares y otros organismos (Turetsky 2003; Cornelissen et al. 2007; Asplund y Wardle 2016). Ambos grupos sirven como hábitat a comunidades de microorganismos y pequeños animales, con lo que influyen en multitud de redes tróficas y rutas de reciclado de nutrientes (Longton 1992; Glime 2014; Asplund y Wardle 2016). En general su paso a las ca- 
denas tróficas es relativamente limitado, tanto por herbivoría como por descomposición, tal vez debido a su abundante producción de metabolitos secundarios (Rundel 1978; Asakawa 2007). No obstante, en zonas boreales, donde son dominantes en cuanto a biomasa, los líquenes constituyen hasta el $50 \%$ de la dieta invernal de los renos (Asplund y Wardle 2016)). En zonas más templadas, aunque en términos de biomasa su contribución es escasa, son muy importantes para algunos animales, en especial gasterópodos (Glime 2006; Asplund y Wardle 2016).

Los líquenes y los briófitos ejercen una importante función de captación de agua y partículas de polvo atmosférico, permitiendo la retención de nutrientes y la formación de sustratos utilizables por plantas vasculares (Cornelissen et al. 2007; Asplund y Wardle 2016)). Su función estabilizadora del ecosistema es particularmente importante en los suelos de zonas áridas y semiáridas (con alta representación en la península ibérica), donde son componentes fundamentales de las llamadas costras biológicas. Estas costras engloban comunidades muy biodiversas de cianobacterias, algas eucariotas, hongos, líquenes, briófitos y protozoos, que colonizan los milímetros más superficiales del sustrato, y juegan un papel fundamental en la formación de la estructura del suelo, prevención de la erosión, lavado de nutrientes, producción de biomasa, y biodisponiblidad de N (Belnap y Lange 2001; Belnap et al. 2008, Maestre et al. 2011). En su conjunto, ambos grupos de organismos se consideran de gran eficacia en la toma de compuestos nitrogenados, sean atmosféricos, del dosel vegetal, o, en menor proporción, del sustrato. Se ha descrito que en ocasiones pueden limitar la disponibilidad de $\mathrm{N}$ para otros organismos (véase, por ejemplo, Turetsky 2003). Sin embargo, en general se considera que, por su asociación facultativa u obligada con cianobacterias fijadoras, y su capacidad de retener nutrientes frente al lavado y la erosión, los briófitos y líquenes suponen generalmente una entrada neta de $\mathrm{N}$ para muchos ecosistemas, en especial en zonas áridas, bosques templados y boreales. Así mismo, destaca su contribución al equilibrio global del ciclo biogeoquímico del N (Glime 2007; Cornelissen et al. 2007).

\section{Impacto del aumento del depósito de $\mathbf{N}$ antropogénico en briófitos y líquenes}

En general, un incremento puntual en las tasas de depósito de $\mathrm{N}$ no suele llegar a causar efectos letales en briófitos ni líquenes. Se ha visto que, por ejemplo, tanto líquenes como musgos pueden tolerar adiciones de hasta $50 \mathrm{~kg} \mathrm{~N}$ ha-1 año-1, al menos a corto plazo (e.g., Gundale et al. 2011, Johansson et al. 2012; OchoaHueso y Manrique 2013). Sin embargo, sí suelen producirse efectos nocivos que se revierten muy lentamente, tal como se ha demostrado en experimentos de trasplante recíproco (Mitchell et al. 2004; Armitage et al. 2011). Debido a las características morfofisiológicas mencionadas anteriormente, los briófitos y líquenes reaccionan sensiblemente a la precipitación de contaminantes atmosféricos. Se han observado alteraciones en el crecimiento, anatomía, fisiología y metabolismo en múltiples especies, así como cambios en la diversidad (alfa y beta) de sus comunidades en diferentes ambientes y áreas geográficas (véanse algunas referencias detalladas más abajo). Dichas alteraciones son visibles normalmente con concentraciones de $10-20 \mathrm{~kg} \mathrm{~N} \mathrm{ha}^{-1} \mathrm{año}^{-1}$, aunque en algunos casos ya hay cambios notables con umbrales críticos bajos, en torno a los 1-3 kg N ha-1 año-1 (Bobbink et al. 2010; Pardo et al. 2011). Las respuestas observables dependen del sistema estudiado, aunque un exceso de $\mathrm{N}$ conlleva, en general: (i) una disminución en la cobertura o biomasa, probablemente por efecto indirecto de mayor competencia con plantas vasculares (e.g., Mitchell et al. 2004; Gundale et al. 2014); (ii) un aumento de N tisular y concentración de pigmentos fotosintéticos y fotoprotectores, que incluso puede no verse limitado por concentraciones atmosféricas crecientes (e.g., Pearce y van der Wal 2002; Wiedermann et al. 2009; Root et al. 2013; Ochoa-Hueso et al. 2014); (iii) una menor fijación de $\mathrm{N}_{2}$, relacionada con un descenso en la densidad de cianobacterias en aquellas especies donde existe asociación simbiótica (e.g., Gundale et al. 2011; Ackerman et al. 2012); (iv) una reducción en la tasa fotosintética, debida a una menor eficiencia del fotosistema II como consecuencia del incremento en la concentración de ciertos pigmentos (e.g., Arróniz-Crespo et al. 2008); y (v) una menor riqueza y abundancia de especies, así como cambios en la composición de las comunidades, normalmente con un predominio especies nitrófilas sobre las oligotróficas (e.g., Wolseley et al. 2006; Davies et al. 2007, Johansson et al. 2012). Estos efectos tienden a ser más notables cuando la contaminación se genera por compuestos reducidos de $\mathrm{N}\left(\mathrm{NH}_{3}\right.$ y $\left.\mathrm{NH}_{4}{ }^{+}\right)$, emisiones típicamente procedentes de prácticas agrícolas (Paulissen et al. 2004; Wiedermann et al. 2009).

Gran parte de nuestro conocimiento sobre el efecto del depósito de $\mathrm{N}$ en estos grupos de plantas se debe a experimentos de simulación en campo y laboratorio mediante la adición de diferentes dosis en condiciones ambientales controladas. Destacan, por ejemplo, los estudios realizados en ecosistemas boreales con los briófitos Hylocomium splendens o Pleurozium schreberi (Gundale et al. 2011, 2014), o aquellos sobre costras biológicas en sistemas áridos o semi-áridos con líquenes de coberturas apreciables sobre el sustrato (e.g., Cladonia foliacea, Diplochistes diacapsis) y especies terí́colas de musgos, como Tortella squarrosa (=Pleurochaete squarrosa) (Ochoa-Hueso y Manrique 2013). En condiciones naturales, también existen cada vez más trabajos que evalúan los efectos biológicos en relación a la distancia al foco de emisión de $\mathrm{N}$ (Ackerman et al. 2012) o comparando diferentes localidades a lo largo de un gradiente geográfico de depósito de N (Flagmeier et al. 2014; Ochoa-Hueso et al. 2014). En ocasiones, se han encontrado resultados contradictorios entre el medio natural y experimental, que se sugiere pueden deberse a las concentraciones muy superiores suministradas en este último caso (Armitage et al. 2012). Además de los estudios empíricos, también se usan modelos de deposición (Stevens et al. 2016) o incluso se han establecido niveles de referencia, como es el caso de los valores de Ellenberg para el N (Delgado y Ederra 2013) o aquellos utilizados característicamente en ciertos grupos de líquenes (Jovan et al. 2012).

Además, la magnitud de estos efectos puede verse condicionada por el clima. Se ha demostrado que la acumulación de compuestos inorgánicos de $\mathrm{N}\left(\mathrm{NO}_{3}{ }^{-}, \mathrm{NH}_{4}{ }^{+}\right)$es promovida por el aumento de temperatura y por el descenso de las precipitaciones, así como por la subsiguiente desaparición de las comunidades de líquenes y briófitos, y de su papel regulador en el ciclo del N (Delgado-Baquerizo et al. 2014; Reed et al. 2012). Otros ejemplos del efecto que ejerce el clima conjuntamente con el depósito de $\mathrm{N}$ incluyen la alteración de la riqueza y composición de especies en diferentes ecosistemas mediterráneos (Jovan y McCune 2005; McMurray et al. 2014), la fijación de $\mathrm{N}_{2}$ en regiones boreales y subárticas (Lett y Michelsen 2014; Whiteley y Gonzalez 2016), y la acumulación de C en turberas de regiones templadas y boreales, así como en costras biológicas de ecosistemas áridos (Granath et al. 2014; UtstølKlein et al. 2015). Cabe resaltar que en condiciones climáticas estresantes, la estacionalidad es un componente importante, pues modula los picos de $\mathrm{N}$ en función de la nitrificación y mineralización que tienen lugar frecuentemente desde finales de primavera hasta mediados de verano (Ochoa-Hueso et al. 2011b). Aparte del clima, las modificaciones del paisaje natural en favor de campos de cultivo y ganadería tienen un papel clave tanto sobre el depósito de $\mathrm{N}$ como sobre la presión de pastoreo, por lo que afectan doblemente a las criptógamas (van der Wal 2003; Pearce et al. 2010; Armitage et al. 2012).

\section{Briófitos y líquenes como biomonitores del depósito de $\mathbf{N}$ antropogénico}

Los efectos negativos causados por la acumulación de compuestos nitrogenados depositados desde la atmósfera han incrementado la preocupación de las sociedades modernas por obtener información acerca de la calidad del medio. La monitorización de los contaminantes atmosféricos, junto con la evaluación de sus efectos en la biosfera, permiten obtener información que los gobiernos pueden utilizar para controlar la contaminación del aire y esta- 
blecer políticas y regulaciones que protejan el medio ambiente. Frente a las técnicas de monitorización tradicional, basadas en análisis físico-químicos estandarizados de precipitación, partículas y gases que se indican en la legislación (Directiva 2008/50/CE; Directiva 2015/1480/CE), se han desarrollado metodologías complementarias como la monitorización biológica o biomonitorización. Estas técnicas permiten detectar los niveles de contaminantes en el medio de un modo sostenible y no requieren un equipamiento costoso que precise de mantenimiento. Además, los análisis son menos complejos, dado que las matrices analizadas presentan concentraciones más elevadas, y son, en general, mucho más económicos. De este modo, la biomonitorización permite crear redes de muestreo más extensas y con una mayor resolución espacio-temporal.

La selección del organismo biomonitor es un paso clave en los estudios de biomonitorización, pues éste debe cumplir ciertos requisitos (Chakrabortty y Paratkar 2006): i) amplia distribución geográfica (el organismo debe ser común en el área de estudio ya sea a nivel local, regional o nacional); ii) accesible, con baja movilidad y disponible durante todo el año; iii) fácilmente identificable y manejable; iv) que proporcione biomasa suficiente para el análisis químico; v) limitada variabilidad biológica (para reducir las diferencias intraespecíficas en la capacidad de acumulación de contaminantes); vi) tolerancia a niveles elevados de contaminantes; vii) que tenga una baja capacidad de regulación interna de los contaminantes; $y$, viii) que la atmósfera sea su fuente principal de nutrientes ( $y$ por lo tanto de contaminantes). Las plantas son probablemente los biomonitores más útiles para el depósito atmosférico de contaminantes, ya que son sésiles, por lo que han de adaptarse a las condiciones locales, y generalmente tienen mayor longevidad que los animales (Bargagli 1998). En particular, los briófitos y los líquenes son ideales para su uso en estudios de biomonitorización gracias a las características fisiológicas y morfológicas mencionadas anteriormente (Fig. 1) . Además, su elevada relación superficie/volumen y la alta capacidad de intercambio catiónico de sus paredes celulares hace que sean capaces de retener contaminantes de forma muy eficiente (Markert et al. 1999).

El empleo de líquenes como bioindicadores se remonta a mediados del siglo XIX. Un buen ejemplo de los trabajos de esta época es el seminal artículo de Nylander (1866) que analizó cua- litativamente los líquenes epífitos de parques y jardines de París y destacó su utilidad como indicadores de la calidad del aire. Pero es a finales del siglo XX cuando se produce un aumento destacable en el número de trabajos que mencionan la contaminación atmosférica y los líquenes. Destaca la publicación de Cislaghi y Nimis (1997) que encontraron una relación muy estrecha entre la biodiversidad de líquenes y el cáncer de pulmón en varones adultos de menos de 55 años y que apuntaba claramente a la contaminación de origen antropogénico del aire por sulfatos y nitratos como factor de riesgo para el cáncer de pulmón. Así, los líquenes se han convertido en uno de los bioindicadores de la calidad del aire más estudiados (Conti y Cecchetti 2001; Fig. 2).

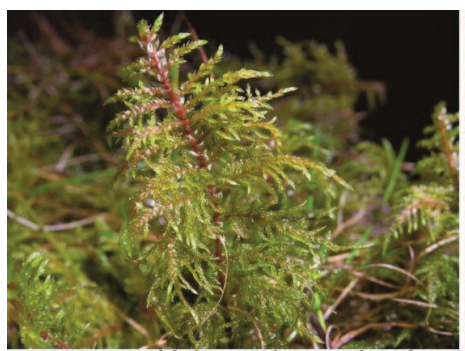

Hylocomium splendens

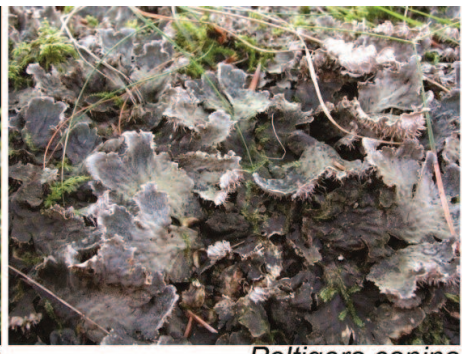

Peltigera canina

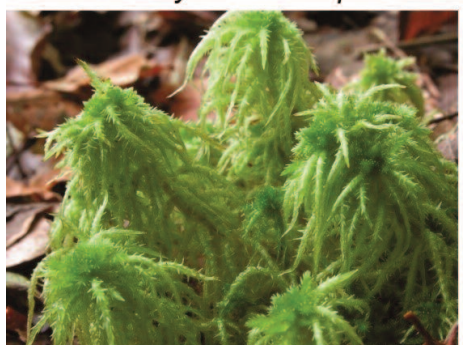

Sphagnum squarrosum

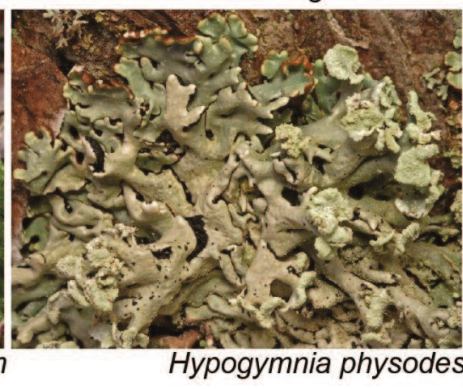

Figura 1. Ejemplos de musgos (derecha) y líquenes (izquierda) frecuentemente empleados en estudios de biomonitorización de la calidad del aire (fotos seleccionadas de la web www.biopix.es).

Figure 1. Examples of mosses (right) and lichens (left) frequently used in air quality biomonitoring studies (all photos were taken from www.biopix.es).

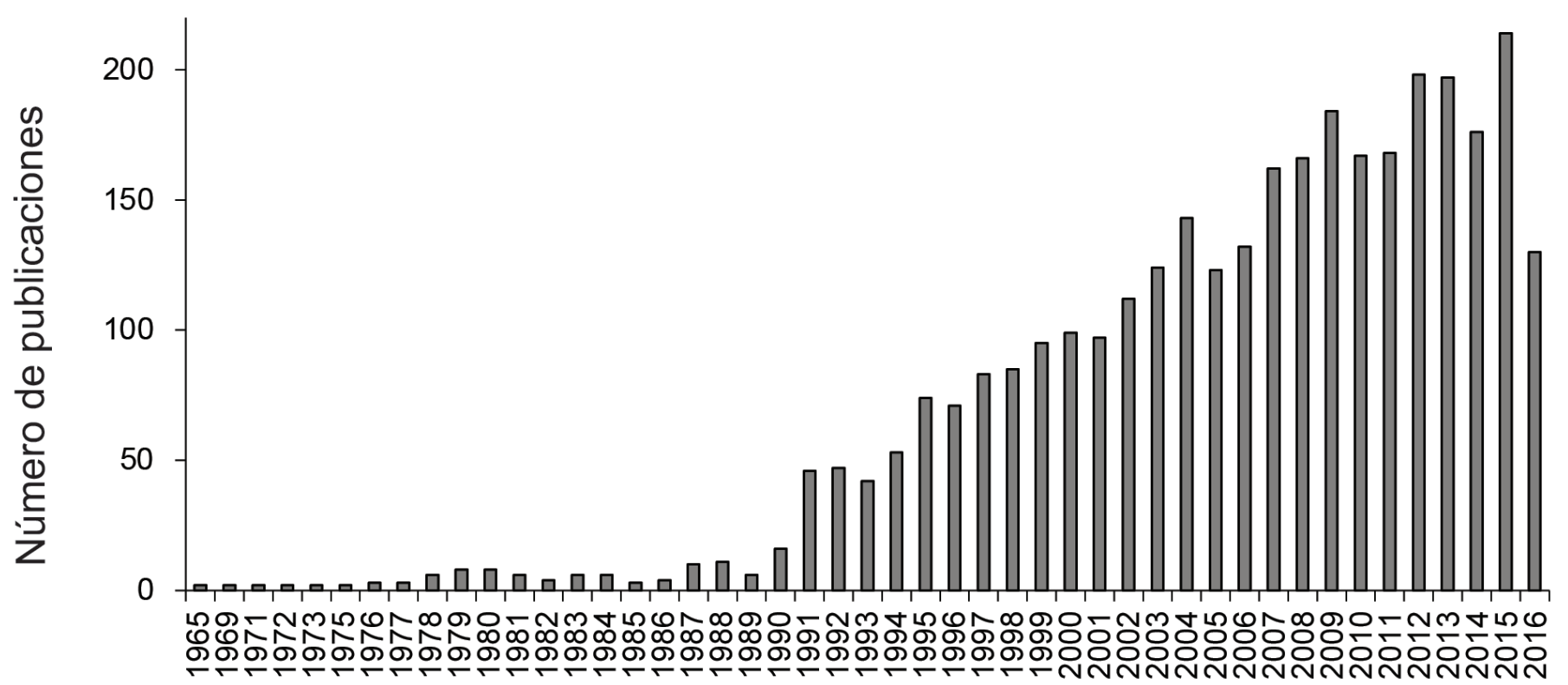

Año

Figura 2. Evolución del número de publicaciones en las que figuran las palabras clave lichen e bioindicadores según ISI WEB of knowledge (motor de búsqueda: lichen (*indic* OR pollution))

Figure 2. Evolution of the number of publications containing lichen and bioindicators as keywords according to ISI WEB of knowledge (searching: lichen (*indic* OR pollution)). 
En cuanto a la relación entre los líquenes y el depósito de N, la mayoría de los trabajos se han centrado en una aproximación basada en el análisis de la biodiversidad y/o los grupos funcionales (Frati et al. 2007; Pinho et al 2009; Aguillaume 2016, Aragón et al 2010, 2016). Por ejemplo, Pinho et al. (2009) analizaron la correspondencia entre diversidad total y diversidad de grupos funcionales en relación a la tolerancia al $\mathrm{N}$ (líquenes nitrófilos vs. oligótrofos) en una zona de elevada actividad ganadera en las inmediaciones de Lisboa y encontraron que la proporción de líquenes oligótrofos frente a nitrófilos cambiaba de forma importante a partir de concentraciones atmosféricas de $\mathrm{NH}_{3}$ por encima de 1 a $2 \mu \mathrm{g} \mathrm{m}^{-3}$. A su vez, Aguillaume (2016), siguiendo una metodología similar, analizó la variación en la diversidad de grupos funcionales (nitrófilos vs. oligótrofos) en encinares mediterráneos y encontró una relación clara entre la deposición de nitrógeno y la diversidad de grupos funcionales pero con unos valores críticos de $2 \mathrm{mg}$ por $\mathrm{m}^{-3}$ mucho más altos que en el caso de Pinho et al. (2009). Por su parte, Aragón et al. (2016) proponen emplear la forma de vida como indicador de la diversidad para simplificar los estudios de diversidad y facilitar su implementación en los sistemas de monitorización. Los resultados de estos trabajos mostraron que, aunque el aumento de especies nitrófilas frente a oligótrofas se correlaciona bien con los niveles de $\mathrm{N}$ atmosférico, también tiene una fuerte relación con la concentración de sulfatos por lo que resulta difícil individualizar el impacto del $\mathrm{N}$ frente a otros contaminantes (Krupa 2003).

El uso de musgos como biomonitores de la calidad del aire comenzó a finales de los 60 con los estudios pioneros de Rühling y Tyler (1968, 1970), dos ecólogos suecos que utilizaron especies terrestres, como Hylocomium splendens, Pleurozium schreberi e Hypnum cupressiforme para determinar la deposición atmosférica de $\mathrm{Pb}$ en Suecia. Desde entonces, el uso de esta técnica se ha extendido e incluso se ha establecido un grupo de trabajo europeo para la biomonitorización de la calidad del aire con musgos terrestres y otras plantas denominado International Cooperative Program on Effects of Air Pollution on Natural Vegetations and Crops (ICP Vegetation). De este modo, los musgos terrestres se han empleado ya con éxito en el desarrollo de mapas de contaminación (Bykowszczenko et al. 2006; Harmens et al. 2013), en la identificación de fuentes de emisión concretas de metales pesados (Fernández et al. 2007), para monitorizar diferentes tipos de compuestos orgánicos (PAHs, PCBs, PCDDs, PCDFS, etc.), y, naturalmente, también para el N (Harmens et al. 2011). La relación de la deposición atmosférica del $\mathrm{N}$ con su contenido en los tejidos de musgos terrestres se estudió por primera vez en el Reino Unido (Pitcairn et al 1995). Desde entonces, otros autores han trabajado en este tema, tanto en experimentos de campo (Pitcairn et al. 2006; Poikolainen et al. 2009; Schröder y Pesch 2010) como de laboratorio (Leith et al. 2005; Solga y Frahm 2006; Salemaa et al. 2008; Stevens et al. 2011). No obstante, otros estudios de biomonitorización del depósito de $\mathrm{N}$ han puesto en evidencia que la relación entre la deposición atmosférica de $\mathrm{N}$ y la acumulación de este elemento en los tejidos del musgo es bastante débil (Pitcairn et al. 2006; Solga et al. 2005; Stevens et al. 2011). Una posible explicación sería la regulación de los niveles de $\mathrm{N}$ tisulares por parte de los propios musgos, dada la importancia que tiene este nutriente en el metabolismo de los briófitos (Koranda et al. 2007; Arróniz-Crespo et al. 2008). Esta regulación podría distorsionar los patrones de deposición de $\mathrm{N}$ obtenidos mediante biomonitorización con musgos terrestres. Por este motivo, la cuantificación de la deposición atmosférica del $\mathrm{N}$ a partir de sus concentraciones totales en el musgo no es posible. Sin embargo, sí que es posible su utilización para identificar zonas expuestas a la contaminación por N (Harmens et al. 2011).

Como una alternativa a la determinación de la concentración total de $\mathrm{N}$ en los tejidos del musgo, más recientemente se ha planteado la posibilidad de determinar el contenido de sus dos isótopos estables $\left({ }^{14} \mathrm{~N}\right.$ y ${ }^{15} \mathrm{~N}$ ) (Solga et al. 2005; Liu et al. 2008; Zechmeister et al. 2008; Varela et al. 2013). Ambos isótopos se encuentran en una proporción constante de forma global en la atmósfera, un $99.63 \%$ de ${ }^{14} \mathrm{~N}$ y un $0.36 \%$ de ${ }^{15} \mathrm{~N}$ (Nier 1950). Diversos trabajos (Freyer 1978; Garten 1992; Heaton et al. 1997) han demostrado que los distintos compuestos de $\mathrm{N}$, tanto oxidados $\left(\mathrm{NO}_{\mathrm{x}}\right)$ como reducidos $\left(\mathrm{NH}_{\mathrm{y}}\right)$, tienen diferentes ratios ${ }^{15} \mathrm{~N} /{ }^{14} \mathrm{~N}$, es decir, diferentes valores en la relación de isótopos estables $\left(\delta^{15} \mathrm{~N}\right)$. Las formas oxidadas del $\mathrm{N}$, que presentan ratios más altas, son emitidas a la atmósfera fundamentalmente por el transporte, la industria y la producción energética (esta última llega a contribuir con hasta un $70 \%$ de estas emisiones; Bragazza et al. 2005). En zonas urbanas, donde este tipo de emisiones son más abundantes, se han registrado valores más altos (más positivos) de $\delta^{15} \mathrm{~N}$ en musgos. Por otro lado, las formas reducidas como $\mathrm{NH}_{\mathrm{y}}$ provienen de actividades agrícolas y uso de fertilizantes, lo que se traduce en valores más bajos (más negativos) de $\delta^{15} \mathrm{~N}$ en musgos (Pearson et al. 2000; Solga et al. 2005; Liu et al. 2008; Zechmeister et al. 2008). En consecuencia, los valores de $\delta^{15} \mathrm{~N}$ en musgos terrestres permitirían la diferenciación de las diversas fuentes de emisión del $\mathrm{N}$ antropogénico.

Así mismo, una aproximación prometedora consiste en el empleo como indicadores del depósito de $\mathrm{N}$ la actividad de enzimas como la fosfomonoestearasa (PME) o la nitrato-reductasa (NR) en líquenes y musgos (Arróniz-Crespo et al. 2008; Ochoa-Hueso et al. 2014; Varela et al. 2016). Este tipo de estudios muestran una alta correlación con el depósito de $\mathrm{N}$ y elevada sensibilidad incluso a concentraciones bajas de contaminante (Ochoa-Hueso et al. 2014), lo que, junto con los análisis de isotopos de $\mathrm{N}$ en briófitos, supone un importante avance en el uso de las criptógamas como bioindicadores.

\section{Caso práctico: uso de musgos terrestres como biomonitores de $\mathbf{N}$ en la península ibérica}

Los estudios de biomonitorización del depósito de $\mathrm{N}$ atmosférico mediante el uso de musgos terrestres realizados hasta la fecha en la península ibérica no son muy numerosos. Autores como González-Miqueo et al. (2009, 2010a, 2010b), Varela et al. (2013), OchoaHueso et al. (2014), Izquieta-Rojano et al. (2016) han intentado evaluar la adecuación de distintas especies de musgos, e.g. Hypnum cupressiforme, Tortella squarrosa (=Pleurochaete squarrosa), o Pseudoscleropodium purum, como biomonitores del depósito de $\mathrm{N}$, a la vez que han buscado posibles tendencias espaciales de dicho elemento a nivel regional. Sus resultados han puesto en evidencia lo expuesto anteriormente acerca de la regulación metabólica a la que se encuentra sometido el $\mathrm{N}$ en briófitos, a lo que se podría sumar la reactividad de los compuestos del $\mathrm{N}$ en la atmósfera y la complejidad de su depósito (e.g. Sutton et al. 2011b), impidiendo que ninguno de los estudios haya podido establecer una clara relación entre el depósito de $\mathrm{N}$ (por existencia de fuentes de contaminación) y su contenido total en el tejido del musgo. Sin embargo, Ochoa-Hueso et al. (2014) sí que han encontrado correlaciones significativas entre la cantidad de $\mathrm{N}$ atmosférico depositado y la actividad de las enzimas fosfomonoesterasa (PME) y nitrato reductasa (NR) en el musgo $T$. squarrosa (=P. squarrosa), así como únicamente de la actividad de la PME en el liquen Cladonia foliacea. Dicha correlación resultó positiva para la PME y negativa para la NR. Así mismo, la relación entre la cantidad de ambas enzimas (PME/NR) en $T$. squarrosa se vio incrementada significativamente sugiriendo un ajuste fisiológico en respuesta a la saturación por $\mathrm{N}$ y el desequilibrio entre la cantidad de fósforo y nitrógeno. Con estos resultados como base, dichos autores han planteado la validez de la relación $\mathrm{PME} / \mathrm{NR}$ como una buena medida fisiológica indicativa de la cantidad de $\mathrm{N}$ atmosférico depositado a escala nacional.

Por su parte, Varela et al. (2013) evaluaron la variación geográfica de la contaminación por depósito de $\mathrm{N}$ mediante la recolección de un total de 165 muestras de $P$. purum alrededor de una fábrica de alúmina-aluminio en las que determinaron las concentraciones totales de $\mathrm{N}$. A partir del foco de emisión de $\mathrm{NO}_{x}$ de la fábrica, y hasta una distancia máxima de $5000 \mathrm{~m}$, establecieron 8 sectores de $30^{\circ}$ de amplitud de donde fueron recogiendo entre 6 y 30 muestras en cada uno de ellos (Fig. 3). Al contrario de lo que cabría esperar por lo obtenido para metales pesados (Fernández et al. 2007), los niveles totales de $\mathrm{N}$ en musgos no reflejaron un gradiente decreciente de la concentración de este elemento con el incre- 


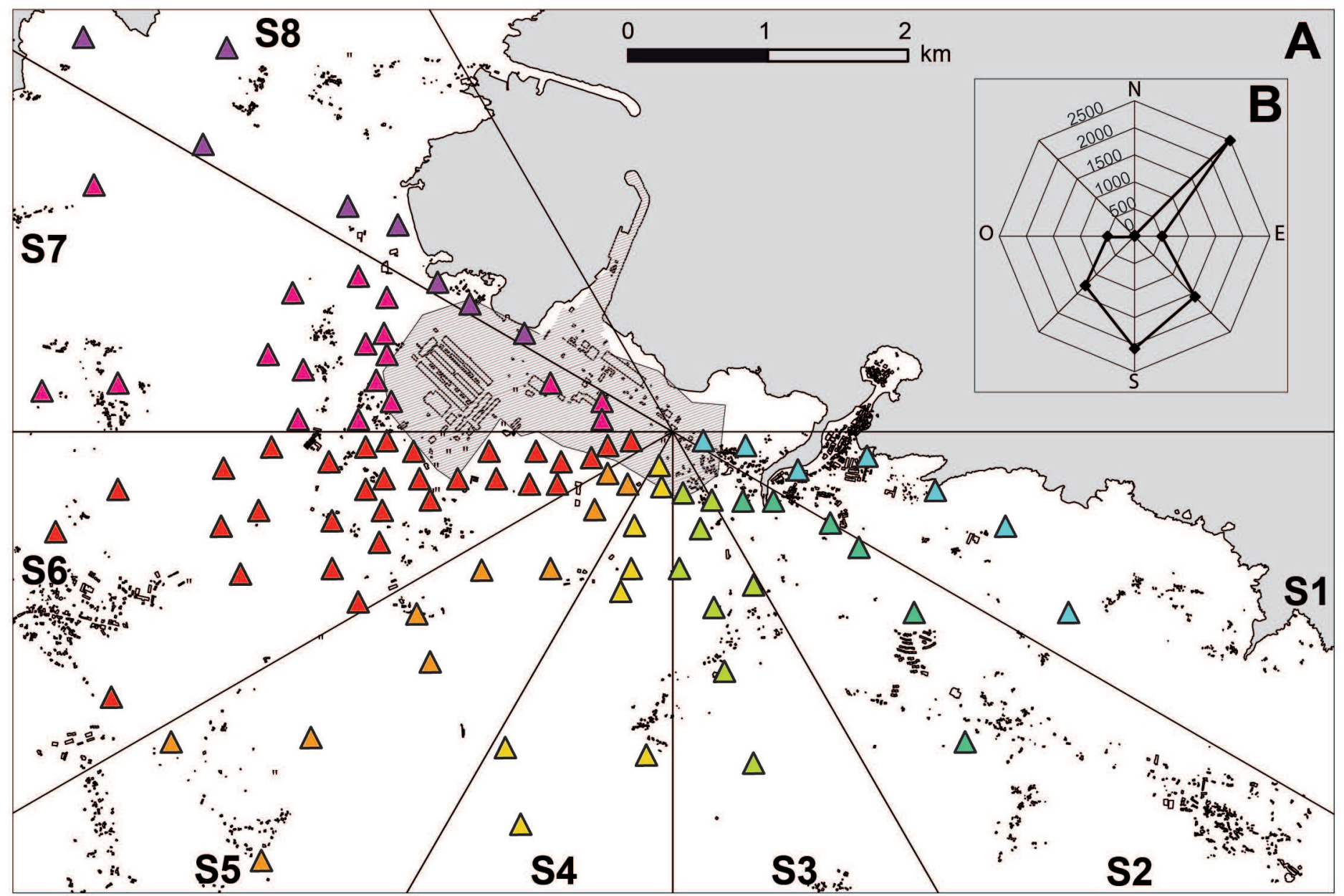

Figura 3. A) Localización de las estaciones de muestreo en los alrededores de la fábrica de alúmina-aluminio en los correspondientes sectores (S1 al S8). B) Rosa de los vientos del área de estudio, la línea gruesa representa las horas de viento.

Figure 3. A) Location of sampling stations around the alumina-aluminium smelter and the assignment of sectors (S1 to S8). B) Wind rose for the study area, the thick line represents hours of wind.

mento de la distancia de las muestras al foco de emisión (Fig. 4) (resultados similares a los obtenidos por González-Miqueo et al. 2010a). Por otro lado, estos mismos autores tampoco encontraron ningún tipo de estructura espacial (i.e. variación espacial sistemática) en las concentraciones de $\mathrm{N}$ total en los tejidos de $P$. purum a escala regional (Galicia, NO de España), ya que sus valores se distribuyeron aleatoriamente por todo el territorio (Fig. 5). No obstante, los resultados de estos mismos análisis obtenidos para la relación isotópica $\delta^{15} \mathrm{~N}$ sí que mostraron un patrón espacial definido, y una buena correspondencia con las actividades económicas predominantes, tal como hemos comentado. Los valores más negativos de $\delta^{15} \mathrm{~N}$, atribuibles a formas reducidas $\left(\mathrm{NH}_{\mathrm{x}}\right)$ se encontraron en la zona central del área de estudio, principalmente ganadera y agrícola, mientras que los valores menos negativos se encontraron en las zonas más pobladas e industrializadas, principalmente: i) la zona costera; ii) la zona sureste, dominada por macizos montañosos pero influida por dos centrales térmicas (fuera de la región de estudio); y iii) la zona norte, sometida a las emisiones de dos centrales térmicas con patrones de depósito de $\mathrm{N}$ previamente estudiados (Méndez et al. 2003). Estos resultados de Varela et al. (2013) en P. purum concuerdan con los encontrados por IzquietaRojano et al. (2016) para T. squarrosa, a los que los valores del $\delta^{15} \mathrm{~N}$ les permitieron identificar las fuentes de contaminación oxidadas y reducidas de $\mathrm{N}$.

La ausencia de correlación entre el $\mathrm{N}$ atmosférico depositado y la concentración en los tejidos del musgo nativo de la mayor parte de los estudios llevados a cabo hasta ahora, ha motivado a Varela et al. (2016) a emplear diversos tipos de trasplantes de musgo $P$. purum para determinar si las distintas formas en las que se encuen- tra el $\mathrm{N}$ en el ambiente (oxidado y reducido) pueden estar influyendo los resultados obtenidos. De hecho, hay varios estudios donde se muestra la preferencia de los briófitos por las formas reducidas de $\mathrm{N}$ frente a las oxidadas, pues su incorporación es menos costosa metabólicamente debido a su alta capacidad de intercambio catiónico (véase Solga y Frahm 2006; o Stevens et al. 2011). De esta manera, Varela et al. (2016) solo encontraron un enriquecimiento del contenido de $\mathrm{N}$ en tejidos de musgos situados alrededor de una granja de vacuno donde la mayor parte del $\mathrm{N}$ ambiental se encuentra en forma reducida. Esto viene a confirmar que no se debería emplear el musgo nativo para monitorizar el $\mathrm{N}$ total depositado, sino restringirlo a los compuestos reducidos.

El desarrollo de herramientas eficaces, económicas y ecológicas para la monitorización de la contaminación atmosférica (metales pesados, nitrógeno, compuestos orgánicos, etc.) constituye un reto importante para la aplicación del conocimiento generado después de muchos años de investigación en biomonitorización. En este sentido, se ha propuesto recientemente una herramienta novedosa desarrollada por un consorcio de grupos de investigación europeos liderados por el grupo de Ecotoxocología de la Universidad de Santiago de Compostela (España). Gracias al proyecto MOSSclone (www.mossclone.eu) financiado por la Unión Europea, estos investigadores han podido crear y patentar un dispositivo que funciona como un sensor de contaminación atmosférica gracias al musgo desvitalizado contenido en el interior de una esfera (la MOSSphere). La exposición del musgo contenido dentro de la MOSSphere durante un periodo de tiempo determinado, y el posterior análisis de su contenido en contaminantes atmosféricos permite caracterizar la calidad del aire en cualquier entorno. 

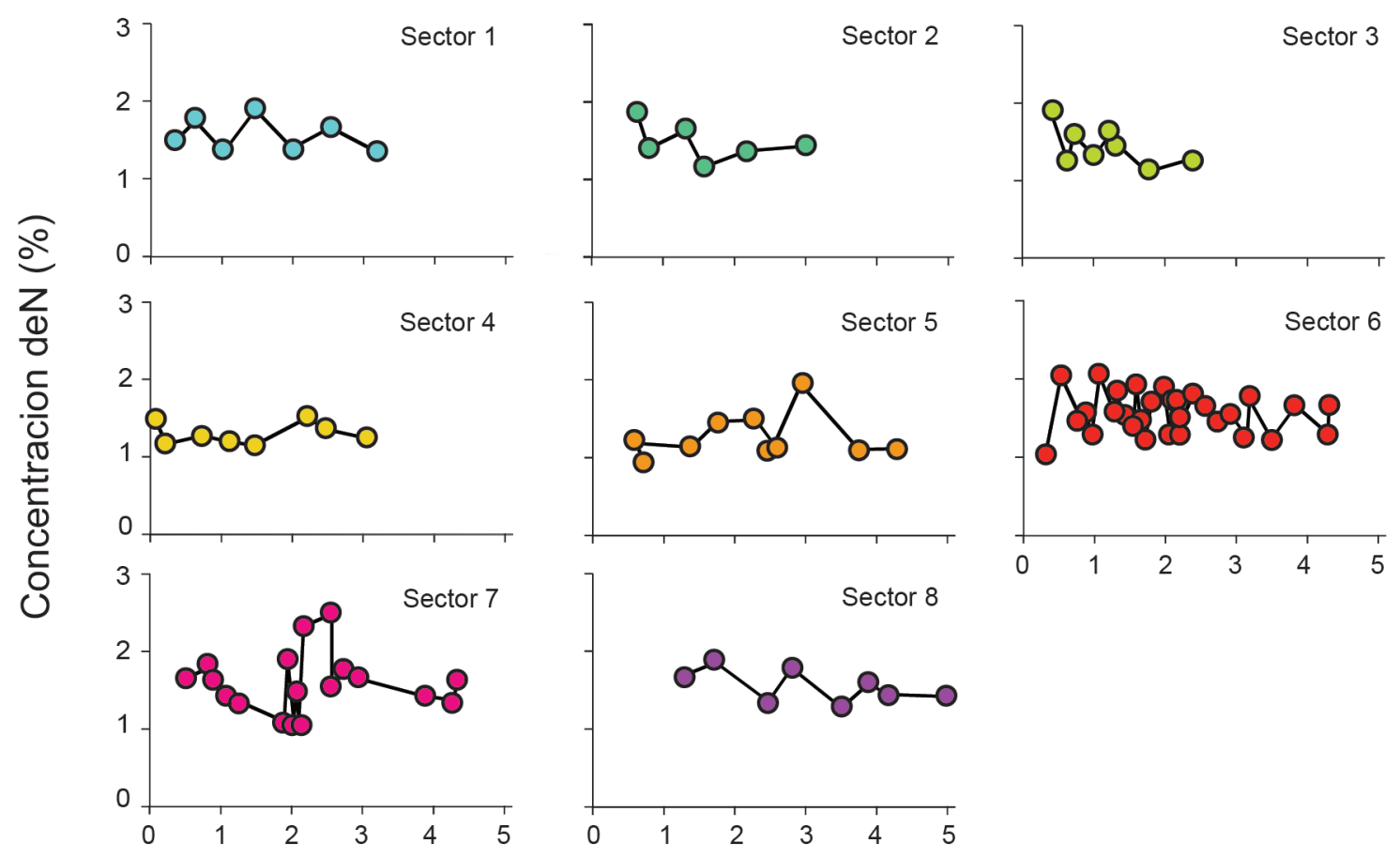

Distancia desde el foco de emisión $(\mathrm{km})$

Figura 4. Relación entre los valores de N (\%) en P. purum y la distancia a la fuente de contaminación de la fábrica de Alúmina-aluminio para cada sector muestreado.

Figure 4. Relation between $N(\%)$ values in P. purum and the distance from the source of contamination in the aluminium smelter for each of the areas sampled.

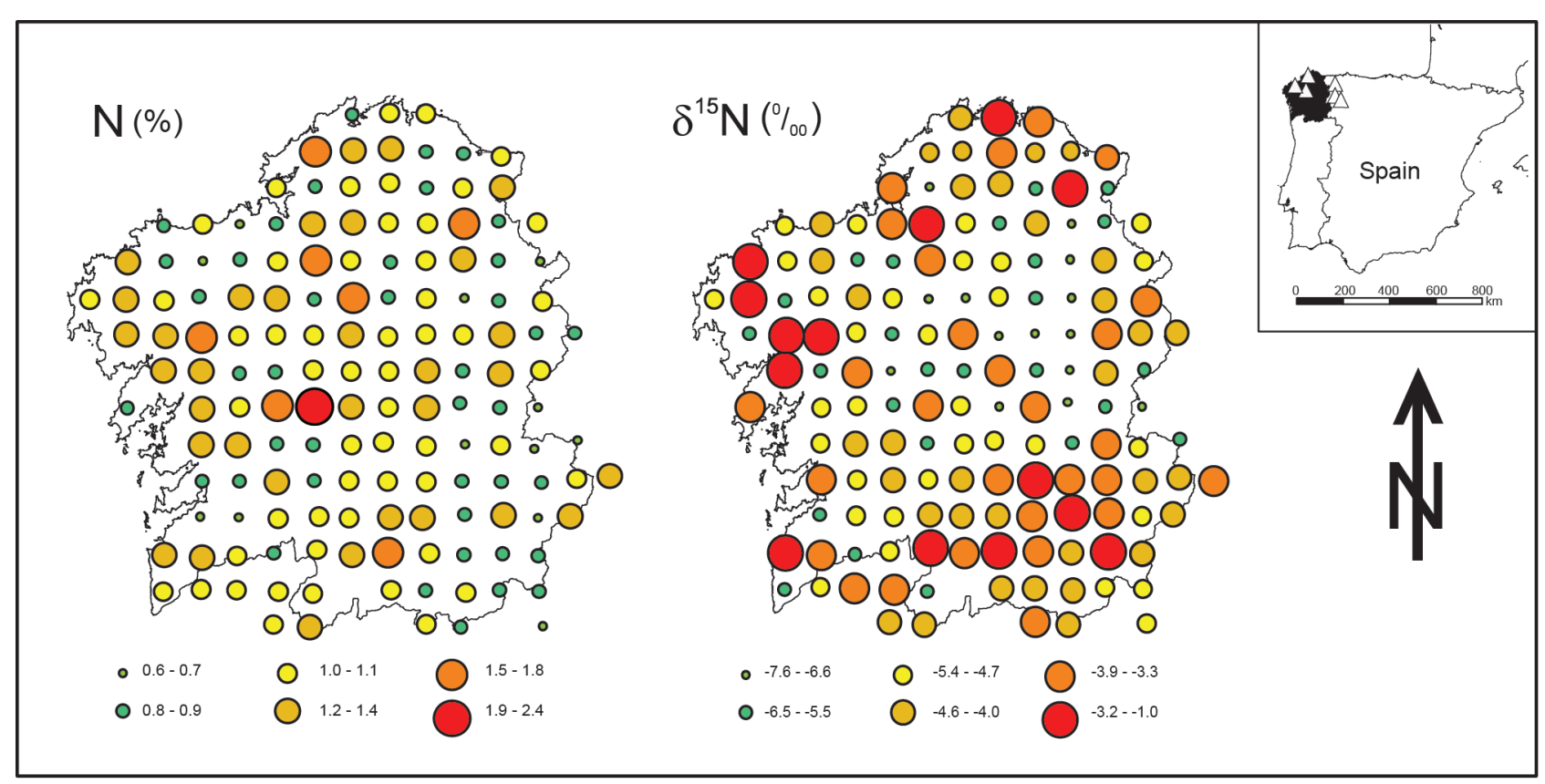

Figura 5. Localización de la zona de estudio (Galicia) y de las centrales térmicas presentes en esta área y alrededores; Concentraciones de $N$ (\%) y de $\delta 15 \mathrm{~N}(\%)$ para P. purum.

Figure 5. Location of study area (Galicia) and the coal-fired power stations in this area and surroundings; Concentrations of $N(\%)$ and $\delta 15 N(\%)$ for $P$. purum.

\section{Conclusiones generales}

Las criptógamas (briófitos y líquenes), a pesar de ser frecuentemente obviadas por su pequeño tamaño con respecto a las plantas vasculares, son organismos fotosintéticos con un papel ecológico fundamental en muchos ecosistemas terrestres (aporte de biomasa en ecosistemas boreales, formación y estabilización del suelo en zonas áridas, formación de hábitat para una gran cantidad de pequeños organismos, etc.), y tienen un rol clave en el ciclo biogeoquímico del $\mathrm{N}$ (fijación del $\mathrm{N}$, incorporación y regulación del disponible para otros organismos). Ciertas especies de líquenes y briófitos son sensibles a los incrementos de la cantidad de $\mathrm{N}$ depositado desde la atmósfera, que puede causar daños tanto a escala individual (crecimiento, anatomía, fisiología y metabolismo) como de comunidad (alteración de la composición/abundancia de especies). Por este motivo, a pesar de la incertidumbre de los modelos actuales, los briófitos y líquenes son buenos organismos para la bioindicación ambiental. 
Por otro lado, las criptógamas presentan una importante capacidad de acumulación del $\mathrm{N}$ ambiental. Si bien los estudios publicados hasta el momento en este campo han indicado que estos organismos presentan limitaciones para estimar la cantidad total depositada de $\mathrm{N}$ atmosférico (debido a su capacidad de regulación metabólica del $\mathrm{N}$ y a la complejidad de los procesos de precipitación y acumulación), sí se ha confirmado que la determinación del $\delta^{15} \mathrm{~N}$ es muy útil para identificar y reconocer las fuentes de emisión de $\mathrm{N}$ (formas reducidas procedentes principalmente de la agricultura y la ganadería y formas oxidadas procedentes de la industria). Además, también se ha demostrado que el estudio de ciertos parámetros fisiológicos (como la relación de enzimas $\mathrm{PME} / \mathrm{NR}$ ) proporciona valiosa información acerca del depósito atmosférico de $\mathrm{N}$.

Por último, a pesar de que la bibliografía científica en cuanto al uso de las criptógamas como bioindicadores/biomonitores de la contaminación por $\mathrm{N}$ en la península ibérica es relativamente limitada, se están realizando avances significativos en el desarrollo de herramientas eficaces, económicas y ecológicas para la monitorización de este elemento.

\section{Agradecimientos}

Las autoras agradecen a Raúl Ochoa-Hueso su invitación a participar en el presente monográfico, así como sus ideas y contribuciones; y a los revisores anónimos, sus comentarios sobre la primera versión del manuscrito.

\section{Referencias}

Ackerman, K., Zackrisson, O., Rousk, J., Jones, D.L., DeLuca, T.H. 2012. $\mathrm{N}_{2}$ fixation in feather mosses is a sensitive indicator of $\mathrm{N}$ deposition in boreal forests. Ecosystems 15: 986-998.

Adams, D.G., Duggan, P.S. 2008. Cyanobacteria-bryophyte symbioses. Journal of Experimental Botany 59: 1047-1058.

Aguillaume, A. 2016. La deposición de nitrógeno en encinares mediterráneos: cargas e indicadores. Ecosistemas 25(2): 110-113.

Alkemade, R., Van Oorschot, M., Miles, L., Nellemann, C., Bakkenes, M., Ten Brink, B. 2009. GLOBIO 3: a framework to investigate options for reducing global terrestrial biodiversity loss. Ecosystems 12: 374-390.

Alpert P., Oliver M.J. 2002. Drying without dying. pp. 3-43 En: Black M., Pritchard H.W. (eds.), Desiccation and survival in plants: drying without dying. CABI Publishing, Wallingford, Reino Unido.

Aragón, G., López, R., Martínez, I. 2010. Effects of Mediterranean dehesa management on epiphytic lichens. Science of the Total Environment 409: 116-122.

Aragón, G., Belinchón, R., Martínez, I., Prieto, M. 2016. A survey method for assessing the richness of epiphytic lichens using growth forms. Ecological Indicators 62: 101-105.

Armitage, H.F., Britton, A.J., Woodin, S.J., van der Wal, R. 2011. Assessing the recovery potential of alpine moss-sedge heath: reciprocal transplants along a nitrogen deposition gradient. Environmental Pollution 159: $140-147$.

Armitage, H.F., Britton, A.J., van der Wal, R., Pearce, I.S.K., Thompson, D.B.A., Woodin, S.J. 2012. Nitrogen deposition enhances moss growth but leads to an overall decline in habitat condition of mountain mosssedge heath. Global Change Biology 18: 290-300.

Arróniz-Crespo, M., Leake, J.R., Horton, P., Phoenix, G.K. 2008. Bryophyte physiological responses to, and recovery from, long-term nitrogen deposition and phosphorus fertilisation in acidic grassland. New Phytologist 180: 864-874.

Asakawa, Y. 2007. Biologically active compounds from bryophytes. Pure and Applied Chemistry 79: 557-580.

Asplund, J., Wardle, D.A. 2016. How lichens impact on terrestrial community and ecosystem properties. Biological Reviews [Doi:10.1111/brv.12305]

Bargagli, R. 1998. Trace elements in terrestrial plants. An ecophysiological approach to biomonitoring and biorecovery. Springer-Verlag, Berlín, Alemania.

Belnap, J., Lange, O.L. 2001. Biological soil crusts: structure, function, and management. Springer-Verlag, Berlín, Alemania.
Belnap, J., Phillips, S.L., Flint, S., Money, J., Caldwell, M. 2008. Global change and biological soil crusts: Effects of ultraviolet augmentation under altered precipitation regimes and nitrogen additions. Global Change Biology 14: 670-686.

Bobbink, R., Hornung, M., Roelofs, J.G.M. 1998. The effects of air-borne nitrogen pollutants on species diversity in natural and semi-natural European vegetation. Journal of Ecology 86: 717-738.

Bobbink, R., Hicks, K., Galloway, J., Spranger, T., Alkemade, R., Ashmore, M., Bustamante, M., Cinderby, S., et al. 2010. Global assessment of nitrogen deposition effects on terrestrial plant diversity: a synthesis. Ecological Applications 20: 30-59.

Bragazza, L., Limpens, J., Gerdol, R., Grosvernier, P., Hájek, M., Hájek, T., Hajkova, P., Hansen I., et al. 2005. Nitrogen concentration and d 15N signature of ombrotrophic Sphagnum mosses at different $\mathrm{N}$ deposition levels in Europe. Global Change Biology 11, 106-114.

Bykowszczenko, N., Baranowska-Bosiacka, I., Bosiacka, B., Kaczmarek, A., Chlubek, D. 2006. Determination of heavy metal concentration in mosses of Słowiński National Park using atomic absorption spectrometry and neutron activation analysis methods. Polish Journal of Environmental Studies 15: 41-46.

Cislaghi, C., Nimis, P.L. 1997. Lichens, air-pollution and lung-cancer. Nature 387 (6632):463-464.

Chakrabortty, S., Paratkar, G.T. 2006. Biomonitoring of trace element air pollution using mosses. Aerosol and Air Quality Research 6(3): 247-258.

Conti, M. E., Cecchetti, G. 2001. Biological monitoring: lichens as bioindicators of air pollution assessment-a review. Environmental Pollution, 114(3):471-492.

Cornelissen, J.H.C., Lang, S.I., Soudzilovskaia, N.A., During, H.J. 2007. Comparative cryptogam ecology: a review of bryophyte and lichen traits that drive biogeochemistry. Annals of Botany 99: 987-1001.

Davies, L. Bates, J.W., Bella, J.N.B., James, P.W., Purvis, O.W. 2007. Diversity and sensitivity of epiphytes to oxides of nitrogen in London. Environmental Pollution 146: 299-310.

De los Ríos, A., Wierzchos, J., Sancho, L.G., Green, T.G.A., Ascaso, C. 2005. Ecology of endolithic lichens colonizing granite in continental Antarctica. The Lichenologist 37(5): 383-395.

Delgado, V., Ederra, A. 2013. Long-term changes (1982-2010) in the bryodiversity of Spanish beech forests assessed by means of Ellenberg indicator values of temperature, nitrogen, light and $\mathrm{pH}$. Biological Conservation 157: 99-107.

Delgado-Baquerizo, M., Maestre, F.T., Escolar, C., Gallardo, A., Ochoa, V., Gozalo, B., Prado-Comesaña, A. 2014. Direct and indirect impacts of climate change on microbial and biocrust communities alter the resistance of the $\mathrm{N}$ cycle in a semiarid grassland. Journal of Ecology 102: 1592-1605.

During, H.J., Van Tooren, B.F. 1990. Bryophyte interactions with other plants. Botanical Journal of the Linnean Society 104: 79-98.

Fernández, J.A., Aboal, J.R., Real, C., Carballeira, A. 2007. A new moss biomonitoring method for detecting sources of small scale pollution. Atmospheric Environment 41: 357-371.

Flagmeier, M., Long, D.G., Genney, D.R., Hollingsworth, P.M., Ross, L.C., Woodin, S.J. 2014. Fifty years of vegetation change in oceanic-montane liverwort-rich heath in Scotland. Plant Ecology and Diversity 7: 457-470.

Frati, L., Santoni, S., Nicolardi, V., Gaggi, C., Brunialti, G., Guttova, A., Gaudino, S., Pati, A., Pirintsos, S.A., Loppi, S. 2007. Lichen biomonitoring of ammonia emission and nitrogen deposition around a pig stockfarm. Environmental Pollution 146: 311-316.

Freyer, H.D. 1978. Seasonal trends of $\mathrm{NH}_{4}{ }^{+}$and $\mathrm{NO}_{3}{ }^{-}$nitrogen isotope composition in rain collected at Jülich, Germany. Tellus 30: 83-92.

Galloway, J.N., Dentener, F.J., Capone, D.G., Boyer, E.W., Howarth, R.W., Seitzinger, S.P., Asner, G.P., Cleveland, C.C., et al. 2004. Nitrogen cycles: past, present and future. Biogeochemistry 70: 153-226.

Galloway, J.N., Townsend, A.R., Erisman, J.W., Bekunda, M., Cai, Z., Freney, J.R., Martinelli, L.A., Seitzinger, S.P., Sutton, M.A. 2008. Transformation of the nitrogen cycle: recent trends, questions and potential solutions. Science 320: 889-892.

Garten Jr, C.T. 1992. Nitrogen isotope composition of ammonium and nitrate in bulk precipitation and forest throughfall. International Journal of Environmental Analytical Chemistry 47: 33-45.

Glime, J.M. 2006. Bryophytes and herbivory. Cryptogamie, Bryologie 27: 191-203. 
Glime, J.M. 2007. Chapter 8-3. Nutrient relations: nitrogen. En: Glime, J. M. (ed.). Bryophyte Ecology. Volume 1. Physiological Ecology. Michigan Technological University and International Association of Bryologists ebook. [Accesible en: http://www.bryoecol.mtu.edu/chapters/8-3NutrientNitrogen.pdf]

Glime, J. M. 2014. The Fauna: A Place to Call Home. Chapt. 1. En: Glime, J. M. (ed.). Bryophyte Ecology. Volume 2. Bryological Interaction. Michigan Technological University and the International Association of Bryologists, e-book. [Accesible en: http://www.bryoecol.mtu.edu/chapters VOL2/1-1AnimalGeneral.pdf].

González-Miqueo, L., Elustondo, D., Lasheras, E., Bermejo, R., Santamaría, J.M. 2009. Spatial trends in heavy metals and nitrogen deposition in Navarra (Northern Spain) based on moss analysis. Journal of Atmospheric Chemistry 62: 59-72.

González-Miqueo, L., Elustondo, D., Lasheras, E., Santamaría, J.M. 2010a. Use of native mosses as biomonitors of heavy metals and nitrogen deposition in the surroundings of two steel works. Chemosphere 78: 965971.

González-Miqueo, L., Elustondo, D., Lasheras, E., Bermejo, R., Santamaría J.M. 2010b. Heavy metal and nitrogen monitoring using moss and topsoil samples in a Pyrenean forest catchment. Water, Air and Soil Pollution 210: 335-346.

Granath, G., Limpens, J., Posch, M., Mücher, S., de Vries, W. 2014. Spatiotemporal trends of nitrogen deposition and climate effects on Sphagnum productivity in European peatlands. Environmental Pollution 187: 73-80.

Gundale, M.J., DeLuca, T.H., Nordin, A. 2011. Bryophytes attenuate anthropogenic nitrogen inputs in boreal forests. Global Change Biology 17 2743-2753.

Gundale, M.J., From, F., Bach, L.H., Nordin, A. 2014. Anthropogenic nitrogen deposition in boreal forests has a minor impact on the global carbon cycle. Global Change Biology 20: 276-286.

Harmens, H., Norris, D.A., Cooper, D.M., Mills, G., Steines, E., Kubin, E., Thöni, L., Aboal, J.R., et al. 2011. Nitrogen concentrations in mosses indicate the spatial distribution of atmospheric nitrogen deposition in Europe. Environmental Pollution 159:2852-2860

Harmens, H., Mills, G., Hayes, F., Norris, D., and the participants of the ICP Vegetation. 2013. Air pollution and vegetation. ICP Vegetation Annual Report 2012/2013. Center for Ecology and Hydrology, Natural Environment Research Council. [Accesible en: http://icpvegetation.ceh.ac.uk/ publications/documents/ICPVegetationannualreport2012-13Final.pdf]

Hauck, M. 2010. Ammonium and nitrate tolerance in lichens. Environmental Pollution 18: 1127-1133.

Heaton, T.H.E., Spiro, B., Robertson, S.M.C. 1997. Potential canopy influences on the isotopic composition of nitrogen and sulphur in atmospheric deposition. Oecologia 109: 600-607.

Izquieta-Rojano, S., Elustondo, D., Ederra, A., Lasheras, E., Santamaría, C., Santamaría, J.M. 2016. Pleurochaete squarrosa (Brid.) Lindb. as an alternative moss species for biomonitoring surveys of heavy metal, nitrogen deposition and $\delta 15 \mathrm{~N}$ signatures in a Mediterranean area. Ecological Indicators, 60:1221-1228.

Johansson, O., Palmqvist, K., Olofsson, J. 2012. Nitrogen deposition drives lichen community changes through differential species responses. Global Change Biology 18: 2626-2635

Jones, E.B.G., Sakayaroj, J., Suetrong, S., Somrithipol, S., Pang, K.L. 2009 Classification of marine Ascomycota, anamorphic taxa and Basidiomycota. Fungal Diversity 35: 1-187.

Jovan, S., McCune, B. 2005. Air-quality bioindication in the greater central valley of California, with epiphytic macrolichen communities. Ecological Applications 15: 1712-1726.

Jovan, S., Riddell, J., Padgett, P.E., Nash, T.A., III. 2012. Eutrophic lichens respond to multiple forms of $\mathrm{N}$ : implications for critical levels and critical loads research. Ecological Applications 22: 1910-1920.

Koranda, M., Kerschbaum, S. Wanek, W., Zechmeister, H., Richter, A. 2007. Physiological responses of bryophytes Thuidium tamariscinum and $\mathrm{Hy}$ locomium splendens to increased nitrogen deposition. Annals of Botany 99: 161-169.

Krab, E., Cornelissen, J.H.C., Lang, S.I., Van Logtestijn, R.S.P. 2008. Amino acid uptake among wide-ranging moss species may contribute to thei strong position in higher-latitude ecosystems. Plant and Soil 304:199208.

Krupa, S.V. 2003. Effects of atmospheric ammonia $\left(\mathrm{NH}_{3}\right)$ on terrestrial vegetation: a review. Environmental Pollution 124: 179-221.
Leith, I.D., Van Dijk, N., Pitcairn, C.E.R., Wolseley, P.A., Whitfield, C.P., Sutton, M.A. 2005. Biomonitoring methods for assessing the impacts of nitrogen pollution: refinement and testing. Report 386. Joint Nature Conservancy Committee. [Accesible en http://jncc.defra.gov.uk/pdf/ jncc386_web_introduction.pdf]

Lett, S., Michelsen, A. 2014. Seasonal variation in nitrogen fixation and effects of climate change in a subarctic heath. Plant and Soil 379: 193204.

Liu, X.-Y., Xiao, H.Y., Liu, C.Q., Li, Y.Y., Xiao, H.W. 2008. Stable carbon and nitrogen isotopes of the moss Haplocladium microphyllum in an urban and a background area (SW China): the role of environmental conditions and atmospheric nitrogen deposition. Atmospheric Environment 42 : 5413-5423.

Longton, R.E. 1992. The role of bryophytes and lichens in terrestrial ecosystems. En: Bates, J.W., Farmer, A.M. (eds.), Bryophytes and lichens in a changing environment, pp. 32-76. Clarendon. Oxford, Reino Unido.

Lucking, R., Hodkinson, B.P., Leavitt, S.D. 2016. The 2016 classification of lichenized fungi in the Ascomycota and Basidiomycota - Approaching one thousand genera. The Bryologist 119: 361-416.

Maestre, F.T., Bowker, M.A., Cantón, Y., Castillo-Monroy, A.P., Cortina, J., Escolar, C., Escudero, A., Lázaro, R., Martínez, I. 2011. Ecology and functional roles of biological soil crusts in semi-arid ecosystems of Spain. Journal of Arid Environments 75: 1282-91.

Markert, B., Wappelhorst, O., Weckert, V., Herpin, U., Siewers, U., Friese, K., Breulmann, G. 1999. The use of bioindicators for monitoring the heavy-metal status of the environment. Journal of Radioanalytical and Nuclear Chemistry 240: 425-429.

Méndez, M.R., Souto, J.A., Casares, J.J., Lucas, T., Carmichael, G.R. 2003. The effect of the limited availability of $\mathrm{H}_{2} \mathrm{O}_{2}$ in the competitive deposition of sulfur and oxidized nitrogen. Chemosphere 53: 1165-1178.

McMurray, J.A., Roberts, D.W., Geiser, L.H. 2014. Epiphytic lichen indication of nitrogen deposition and climate in the northern rocky mountains, USA Ecological Indicators 49: 154-161.

Medina, N.G., Albertos, B., Lara, F., Mazimpaka, V., Garilleti, R., Draper, D., Hortal, J. 2013. Species richness of epiphytic bryophytes: drivers across scales on the edge of the Mediterranean. Ecography 36: 1-14.

Mitchell, E.A.D., Gilbert, D., Buttler, A., Grosvernier, P., Amblard, C., Gobat, J.-M. 2003. Structure of microbial communities in Sphagnum peatlands and effect of atmospheric carbon dioxide enrichment. Microbial Ecology 46: 187-199.

Mitchell, R.J., Sutton, M.A., Truscott, A.-M., Leith, I.D., Cape, J.N., Pitcairn, C.E.R, Van Dijk, N. 2004. Growth and tissue nitrogen of epiphytic Atlantic bryophytes: effects of increased and decreased atmospheric $\mathrm{N}$ deposition. Functional Ecology 18: 322-329.

Nier, A. O. 1950. A redetermination of the relative abundances of the isotopes of carbon, nitrogen, oxygen, argon and potassium. Physical Review 77: 789-793.

Nimis, P. L., Pinna, D., Salvadori, O. 1992. Licheni e conservazione dei monumenti. Cooperativa Libraria Universitaria Editrice, Bolonia, Italia.

Nylander, M.W. 1866. Les lichens du Jardin du Luxembourg. Bulletin de la Société botanique de France 13: 364-371.

Ochoa-Hueso, R., Hernández, R.R., Pueyo, J.J., Manrique, E. 2011a. Spatial distribution and physiology of biological soil crusts from semi-arid central Spain are related to soil chemistry and shrub cover. Soil Biology and Biochemistry 43: 1894-1901.

Ochoa-Hueso, R., Allen, E. B., Branquinho, C., Cruz, C., Dias, T., Fenn, M. E., et al. 2011b. Nitrogen deposition effects on Mediterranean-type ecosystems: an ecological assessment. Environmental Pollution 159: 2265-2279.

Ochoa-Hueso, R., Arróniz-Crespo, M., Bowker, M.A., Maestre, F.T., PérezCorona, M.E., Theobald, M.R., Vivanco, M.G., Manrique, E. 2014. Biogeochemical indicators of elevated nitrogen deposition in semiarid Mediterranean ecosystems. Environmental Monitoring and Assessment 186: 5831-5842

Ochoa-Hueso, R., Manrique, E. 2013. Effects of nitrogen deposition on growth and physiology of Pleurochaete squarrosa (Brid.) Lindb., a terricolous moss from Mediterranean ecosystems. Water, Air, and Soil Pollution 224: 1-14.

Palmqvist, K., Dahlman, L., Valladares, F., Tehler, A., Sancho, L.G., Mattsson, J.-E. 2002. $\mathrm{CO}_{2}$ exchange and thallus nitrogen across 75 contrasting lichen associations from different climate zones. Oecologia 133: 295-306. 
Pardo, L.H., Fenn, M.E., Goodale, C.L., Geiser, L.H., Driscoll, C.T., Allen, E.B., et al. 2011. Effects of nitrogen deposition and empirical nitrogen critical loads for ecoregions of the United States. Ecological Applications 21: 3049-3082.

Paulissen, M.P.C.P., Van Der Ven, P.J.M., Dees, A.J., Bobbink, R. 2004. Differential effects of nitrate and ammonium on three fen bryophyte species in relation to pollutant nitrogen input. New Phytologist 164: 451-458.

Pearce, I.S.K., van der Wal, R. 2002. Effects of nitrogen deposition on growth and survival of montane Racomitrium lanuginosum heath. Biological Conservation 104: 83-89.

Pearce, I.S.K., Britton, A.J., Armitage, H.F., Jones, B. 2010. Additive impacts of nitrogen deposition and grazing on a mountain moss-sedge heath Botanica Helvetica 120: 129-137.

Pearson, J., Wells, D.M., Seller, K.J., Bennett, A., Soares, A., Woodall, J. Ingrouille, M.J. 2000. Traffic exposure increases natural ${ }^{15} \mathrm{~N}$ and heavy metal concentrations in mosses. 2000. New Phytologist 147: 317-326.

Pinho, P., Branquinho, C., Cruz, C., Tang, Y.S., Dias, T., Rosa, A.P., Máguas, Martins-Loução, M.-A. Sutton, M.A. 2009. Assessment of Critical Levels of Atmospheric Ammonia for Lichen Diversity in Cork-Oak Woodland, Portugal. - En: Sutton, M. A., Reis, S., Baker, S. (eds), Atmospheric Ammonia, pp. 109-119. Springer Netherlands. Dordrecht, Países Bajos.

Pitcairn, C.E.R., Fowler, D., Grace, J. 1995. Deposition of fixed atmospheric nitrogen and foliar nitrogen content of bryophytes and Calluna vulgaris (L.) Hull. Environmental Pollution 99: 193-205.

Pitcairn, C., Fowler, D., Leith, I., Sheppard, L., Tang, S., Sutton, M., Famulari, D. 2006. Diagnostic indicators of elevated nitrogen deposition. Environmental Pollution 144: 941-950.

Poikolainen, J., Piispanen, J., Karhu, J., Kubin, E. 2009. Long-term changes in nitrogen deposition in Finland (1990-2006) monitored using the moss Hylocomium splendens. Environmental Pollution 157: 3091-3097.

Purvis, W. 2000. Lichens. Smithsonian Institution Press, Washington, DC. Estados Unidos.

Qiu, Y.-L., Li, L., Wang, B., Chen, Z., Knoop, V., Groth-Malonek, M., Dombrovska, O., Lee, J., et al. 2006. The deepest divergences in land plants inferred from phylogenomic evidence. Proceedings of the National Academy of Sciences 103(42): 15511-15516.

Reed, S.C., Coe, K.K., Sparks, J.P., Housman, D.C., Zelikova, T.J., Belnap, J. 2012. Changes to dryland rainfall result in rapid moss mortality and altered soil fertility. Nature Climate Change 2: 752-755.

Root, H.T., Geiser, L.H., Fenn, M.E., Jovan, S., Hutten, M.A., Ahuja, S., Dillman, K., Schirokauer, D., et al. 2013. A simple tool for estimating throughfall nitrogen deposition in forests of western North America using lichens. Forest Ecology and Management 306: 1-8.

Rühling, Å., Tyler, G. 1968. An ecological approach to the lead problem. Botaniska Notiser 122: 248-342.

Rühling, A., Tyler, G. 1970. Sorption and retention of heavy metals in the woodland moss Hylocomium splendens (Hedw.) Br et Sch. Oikos 21: 9297.

Rundel, P.W. 1978. The ecological role of secondary lichen substances. Biochemical Systematics and Ecology 6: 157-170.

Salemaa, M., Mäkipää, R., Oksanen, J. 2008. Differences in the growth response of three bryophyte species to nitrogen. Environmental Pollution 152: 82-91.

Schröder, W., Pesch, R. 2010. Long-term monitoring of the metal accumulation in forests measured by use of the moss technique. European Journal of Forest Research 129: 475-488.

Shaw, A. J., Szövényi, P., Shaw, B. 2011. Bryophyte diversity and evolution: windows into the early evolution of land plants. American Journal of Botany 98: 352-369.

Sheppard, L.J., Leith, I.D., Mizunuma, T., Cape, J.N., Crossley, A., Leeson, S., Sutton, M.A., Fowler, D., Dijk, N. 2011. Dry deposition of ammonia gas drives species change faster than wet deposition of ammonium ions: evidence from a long-term field manipulation. Global Change Biology 17(12): 3589-3607.

Solga, A., Burkhardt, J., Zechmeister, H.G., Frahm, J.P. 2005. Nitrogen content, ${ }^{15} \mathrm{~N}$ natural abundance and biomass of the two pleurocarpous mosses Pleurozium schreberi (Brid.) Mitt. and Scleropodium purum (Hedw.) Limpr. in relation to atmospheric nitrogen deposition. Environmental Pollution 134: 465-473.

Solga, A., Frahm, J.P. 2006. Nitrogen accumulation by six pleurocarpous moss species and their suitability for monitoring nitrogen deposition. Journal of Bryology 28: 46-52.

Spribille, T., Tuovinen, V., Resl, P., Vanderpool, D., Wolinski, H., Aime, M.C., Schneider, K., Stabentheiner, E., et al. 2016. Basidiomycete yeasts in the cortex of ascomycete macrolichens. Science 353 (6298): 488-492.

Stevens, C.J., Duprè, C., Dorland, E., Gaudnik, C., Gowing, D.J.G., Bleeker, A., Diekmann, M., Alard, D., et al. 2011. The impact of nitrogen deposition on acid grasslands in the Atlantic region of Europe. Environmental Pollution 15: 2243-2250.

Stevens, C.J., Payne, R.J., Kimberley, A., Smart, S.M. 2016. How will the semi-natural vegetation of the UK have changed by 2030 given likely changes in nitrogen deposition? Environmental Pollution 208: 879-889.

Sutton, M.A., Oenema, O., Erisman, J.W., Leip, A., van Grinsven, H., Winiwarter, W. 2011a. Too much of a good thing. Nature 472: 159-161.

Sutton, M. A., Howard,C. M., Erisman, J. W., Billen, G., Bleeker, A., Grennfelt, P., van Grinsven, H., Grizzetti, B. 2011b. The European Nitrogen assessment. Cambridge University Press. Cambridge, Reino Unido.

Turetsky, M.R. 2003. The role of bryophytes in carbon and nitrogen cycling The Bryologist 106: 395-409.

Utstøl-Klein, S., Halvorsen, R., Ohlson, M. 2015. Increase in carbon accumulation in a boreal peatland following a period of wetter climate and long-term decrease in nitrogen deposition. New Phytologist 206: 12381246.

Van der Wal, R., Pearce, I.S.K., Brooker, R., Scott, D., Welch, D., Woodin, S.J. 2003. Interplay between nitrogen deposition and grazing causes habitat degradation. Ecology Letters 6: 141-146.

Varela, Z., Carballeira, A., Fernández, J.A., Aboal, J.R. 2013. On the use of epigaeic mosses to biomonitor atmospheric deposition of nitrogen. Archives of Environmental Contamination and Toxicology 64: 562-572.

Varela, Z, García-Seoane, R, Arróniz-Crespo, M., Carballeira, A., Fernández, J.A., Aboal, J. R. 2016. Evaluation of the use of moss transplants (Pseudoscleropodium purum) for biomonitoring different forms of air pollutant nitrogen compounds. Environmental Pollution 213: 841-849.

Vitousek, P.M., Field, C.B. 2001. Input/output balances and nitrogen limitation in terrestrial ecosystems. En: Schulze E.-D. et al. (eds.)., Global Biogeochemical Cycles in the Climate System, pp. 217-225. Academic Press. San Diego, Estados Unidos.

Whiteley, J.A., Gonzalez, A. 2016. Biotic nitrogen fixation in the bryosphere is inhibited more by drought than warming. Oecologia 181: 1243-1258.

Wiedermann, M.M., Gunnarsson, U., Ericson, L., Nordin, A. 2009. Ecophysiological adjustment of two Sphagnum species in response to anthropogenic nitrogen deposition. New Phytologist 181: 208-217.

Wolseley, P.A., James, P.W., Theobald, M.R., Sutton, M.A. 2006. Detecting changes in epiphytic lichen communities at sites affected by atmospheric ammonia from agricultural sources. The Lichenologist 38: 161 176.

Zechmeister, H.G., Richter, A., Smidt, S., Hohenwallner, D., Roder, I., Maringer, S., Wanek, W. 2008. Total nitrogen content and $\delta^{15} \mathrm{~N}$ signatures in moss tissue: indicative value for nitrogen deposition patterns and source allocation on a nationwide scale. Environmental Science and Technology 42(23): 8661-8667.

Zedda, L., Rambold, G. 2015. The diversity of lichenised fungi: 7. Ecosystem functions and ecosystem services. En: Upreti, D.K., Divakar, P.K, Shukla, V., Bajpai, R. (eds.), Recent advances in Lichenology, pp. 121145. Springer. N. Delhi, India. 